\title{
Solving Fractional Damped Burgers' Equation Approximately by Using The Sumudu Transform (ST) Method
}

\author{
Wurood Riyadh Abd AL- Hussein
}

\author{
Rand Muhaned Fawzi
}

Department of Accounting, Al-Esraa University College, Baghdad, Iraq.

*Corresponding author: wowomath91@yahoo.com, rand moh88@yahoo.com

"ORCID ID: https://orcid.org/0000-0002-9964-9453, https://orcid.org/0000-0003-0106-6688

Received 11/7/2019, Accepted 10/6/2020, Published 30/3/2021

This work is licensed under a Creative Commons Attribution 4.0 International License.

\begin{abstract}
In this work, the fractional damped Burger's equation (FDBE) formula $D_{t}^{\alpha} u+u u_{x}-u_{x x}+\lambda u=0$, $x \in \mathbb{R}, t>0, \lambda>0$ is solved by Sumudu transform (ST) method. The purpose of the method is based on the application of ST method to nonlinear fractional partial differential equations (NLFPDE). Same initial conditions( IC) are selected to clarify this method. Also, the dependability of this method is given by several examples. In other words, some approximate solutions were gotten successfully. Finally, the curve 3D program is used for plotting the figures.
\end{abstract}

Keywords: Caputo derivative, Fractional calculus, Fractional damped Burger's equation, Sumudu transform.

\section{Introduction:}

The NLFPDE is shown in many departments of Engineering, Physics and Applied Mathematical (1). Also,in several phenomena such as elastic viscosity, electromagnetic, material science, acoustic and electrical chemistry. In general, there is no way to give a precise solution to fractional differential equations (FDE) . Only approximate solutions can be inferred. Burger's equation (BE) is the characteristic equation used for many physically interesting problems and for convection-diffusion Phenomena. It is widely applied on water designing in unsaturated oil, soil dynamic in water, static flow difficulties, confusion and strong propagation, cosmogony and earthquakes(2). The BE was initially proposed by Bateman H.,(1915) (3) while modeling the weak viscous liquid motion. The non-linear partial differential equation was published in 1948 by Burgers J. M. (4) It is similar to the onedimensional Navier-stokes equation except for the term stress. Later he studied the B E by Cole J., 1951 (5) who has given a theoretic solution based on Fourir series analysis, using the initial and border conditions. Gorguiis in 2005 (6) compared the transformation of Cole - Hopf and Decomposition method to solve B E. The time_fractional BE was firstly treated by Momani
S., (2006) (7) by the Adomian decomposition method. Biazar and Aminikhah in 2009 (8) solved $\mathrm{BE}$ by using the variation literation technique to find the Approximate solution, whichever is best than ADM.In (2014) Saad and Munaa (9) used Bernoulli equation to solve BE. The ST method is an important transform method that was suggested in early 1993 by Watugala (10). It is a gadget for solving many kinds of PDEs in different fields of engineering and sciences. Different methods are common with a ST method as the homotopy analysis technique and the ST method Rathore, Kumr, Singh and Guptaa (2012) (11). Kurulay M. in (2010) (12) solved the space time fractional BE using differential transform method. Mohammad J. and Bashir A. (2013)(13) used laplace inversion technique method for solveing the fractional partial differential equations (FPDEs)

In this study the fractional Damped Burgers' equation were solved to get approximately solution by using the Sumudu Transform (ST) method.

This study is organized as follows: section two contains some definitions. Section three contains solution by ST method. Section four contains the conclusions. 


\section{Definitions}

Now, some definitions were stated of the fractional calculus and ST that will be used in this work.

\section{Definition ( 1)}

The real function $f(x), x>0$, is said to be in the space $C_{\mu}, \mu \in \mathbb{R}$, if there exists a real number $\mathrm{p}$ (> $\mu)$, as $f(x)=x^{p} f_{1}(x)$, where $f_{1}(x) \in \mathrm{C}[0, \infty)$, and it is said to be in the space $C_{\mu}^{l}$ if $f^{(l)}(x) \in C_{\mu}, l$ $\in \mathbb{N}_{0}=\mathbb{N} \cup\{0\} .(14)$

\section{Definition (2)}

On the (left side) is Riemann Liouville fractional integral of order $\alpha \geq 0$, of a function $f(x) \in C_{\mu}, \mu \geq$ -1 is known as:

$$
I^{\alpha} f(x)=\frac{1}{\Gamma(\alpha)} \int_{0}^{x}(x-t)^{\alpha-1} f(t) d t, \quad x>0
$$

$$
I^{0} f(x)=f(x)
$$

Some properties of $J^{\alpha}$ can be found for $f \in C_{\mu}, \mu \geq$ $-1, \alpha, \beta \geq 0$ and $\delta>-1$, the following expressions are obtained;

1. $I^{\alpha} I^{\beta} f(x)=I^{\alpha+\beta} f(x)=I^{\beta} I^{\alpha} f(x)$;

2. $I^{\alpha} C=\frac{C}{\Gamma(\alpha+1)} x^{\alpha}, C$ is constant ;

3. $I^{\alpha} x^{\delta}=\frac{\Gamma(\delta+1)}{\Gamma(\alpha+\delta+1)} x^{\alpha+\delta}$.

\section{Definition (3)}

The Caputo fractional derivative (CFD)of $f(x)$ is known as;

$D_{*}^{\alpha} f(x)=I^{r-\alpha} D^{r} f(x)=\frac{1}{\Gamma(r-\alpha)} \int_{0}^{x}(x-$

$t)^{r-\alpha-1} f^{(r)}(t) d t, \alpha>0$

For $\mathrm{r}-1<\alpha \leq \mathrm{r}, \mathrm{r} \in \mathbb{N}, x>0$ and the Gamma function is $\Gamma($.$) . (14)$

\section{Definition (4)}

Let $r$ be the smallest integer that override $\alpha$, the CFD of a function $\mathrm{u}(\mathrm{x}, \mathrm{t})$ of order $\alpha>0$ is defined by;

$D_{* t}^{\alpha} u(x, t)=\frac{\partial^{\alpha} u(x, t)}{\partial t^{\alpha}}=$

$\left\{\begin{array}{cc}\frac{1}{\Gamma(r-\alpha)} \int_{0}^{t}(t-\tau)^{r-\alpha-1} \frac{\partial^{r} u(x, \tau)}{\partial \tau^{r}} d \tau, & r-1<\alpha<r \\ \frac{\partial^{r} u(x, t)}{\partial t^{r}}, & \alpha=r \in \mathbb{N}\end{array}\right.$

Which satisfies the following :

1. $D_{*}^{\alpha} C=0, C$ is constant;

2. $D_{*}^{\alpha} x^{\delta}=\frac{\Gamma(\delta+1)}{\Gamma(\delta-\alpha+1)} x^{\delta-\alpha}, x>0, \quad \delta>-1$;

3. $D_{*}^{\alpha}\left(\sum_{i=0}^{m} c_{i} f_{i}(x, t)\right)=\sum_{i=0}^{m} c_{i} D^{\alpha} f_{i}(x, t)$, where $c_{0}, c_{1}, \ldots, c_{m}$ are constants. (14)

\section{Lemma 1}

If $\mathrm{r}-1<\alpha \leq \mathrm{r}, f \in C_{\mu}^{r}, \mathrm{r} \in \mathbb{N}$ and $\delta \geq-1$, so; $D_{*}^{\alpha} I^{\alpha} f(x)=f(x)$ and
$I^{\alpha} D_{*}^{\alpha} f(x)=f(x)-\sum_{k=0}^{r-1} f^{k}\left(0^{+}\right) \frac{x^{k}}{k !}$, where $x>$ 0. (14)

\section{Definition (5)}

ST over the following set of functions ,

$A=\left\{f(\mathrm{t})\left|\exists W, \mathrm{v}_{1}, \mathrm{v}_{2}>0,\right| \mathrm{f}(t) \mid<\mathrm{e}^{\frac{|\mathrm{t}|}{v_{j}}}\right.$, if $\mathrm{t} \in$ $\left.(-1)^{\mathbf{j}} \times[0, \infty)\right\}$

Now, define

Is defined as, for $u \in\left(-v_{1}, v_{2}\right)$, then;

$\mathbb{S}[f(t)]=Q(u)=\int_{0}^{\infty} f(u \mathrm{t}) \mathrm{e}^{-\mathrm{t}} d t=$

$\int_{0}^{\infty} \frac{1}{u} f(t) e^{\frac{-t}{u}} d t$

Some properties of the ST:

1. $\mathbb{S}[1]=1$

2. $\mathbb{S}\left[\frac{t^{r}}{\Gamma(r+1)}\right]=u^{r}, \mathrm{r}>0$;

3. $\mathbb{S}\left[e^{a t}\right]=\frac{1}{1-a u}$;

4. $\mathbb{S}[\alpha f(x) \pm \beta \mathrm{g}(x)]=\alpha \mathbb{S}[f(x)] \pm \beta \mathbb{S}[\mathrm{g}(x)]$. $(15,16)$

\section{Definition (6)}

Let $Q(u)$ be the ST of $f(t)$, as follows

1. $Q(1 / \mathrm{s}) / \mathrm{s}$, is a meromorphic function, with singularities $\operatorname{Re}(\mathrm{s})<\delta$,

2. There exists a circular region $\Gamma$ with radius $\mathrm{R}$ and positive constants, $\mathrm{W}$ and $\mathrm{N}$ with

So the function $f(\mathrm{t})$ is;

$$
\left|\frac{Q(1 / s)}{s}\right|<\mathrm{W} R^{-N}
$$

$f(t)=\mathbb{S}^{-1}[Q(t)]=\frac{1}{2 \pi i} \int_{\gamma-i \infty}^{\gamma+\infty} e^{s t} Q\left(\frac{1}{s}\right) \frac{d s}{s} \quad=$ $\sum$ residues $\left[e^{s t} \frac{Q(1 / s)}{s}\right]$. (15)

Lemma 2. $(1,15,16)$

The ST $\mathbb{S}[f(t)]$ of the Caputo fractional integral (CFI) is defined;

$$
\mathbb{S} \quad\left[D_{t}^{\alpha} f(t)\right]=\frac{G(u)}{u^{\alpha}}-\sum_{k=0}^{n-1} \frac{f^{(k)}(0)}{u^{\alpha-k}}, \quad \text { where }
$$

$G(u)=\mathbb{S}[f(t)]$

Then, that can be easily understood

$$
\mathbb{S}\left[D_{t}^{\alpha} f(x, \mathrm{t})\right]=\frac{\mathbb{S}[f(x, t)]}{u^{\alpha}}-\sum_{k=0}^{n-1} \frac{f^{(k)}(x, 0)}{u^{\alpha-k}}, n-1<\alpha \leq
$$

$n$

\section{Solution by ST method $(16,17)$}

To clarify the baser clue of Sumudu Adomian decomposition technique, the following NLFPDE of the form:

$D_{t}^{\alpha} u(x, t)=\boldsymbol{L} u(x, t)+\boldsymbol{N} u(x, t)+g(x, t)$

(7)

With $n-1<\alpha \leq n$, ratio to the IC 


$$
\frac{\partial^{(\alpha)} u(x, 0)}{\partial t^{\alpha}}=\left.u^{(\alpha)}(x, 0)\right|_{t=0}=f(x), \quad \alpha=0,1,
$$

$2, \ldots, n-1$.

and $D_{t}^{\alpha} u(x, t)$ is the CFD, $g(x, t)$ is the exporter term, $\boldsymbol{L}$ is the linear factor and $\boldsymbol{N}$ is the general nonlinear operator. Now, the ST is fitted on two sides of equation (7)

$$
\mathbb{S}\left[D_{t}^{\alpha} u(x, t)\right]=\quad \mathbb{S}[\boldsymbol{L}(x, t)+\boldsymbol{N} u(x, t)+
$$$$
g(x, t)]
$$

Using the ST property (6) and substituting into (9) to get:

$$
u^{-\alpha} \mathbb{S}[u(x, t)]-\sum_{k=0}^{m-1} u^{-(\alpha-k)} u^{(k)}(x, 0)=
$$$$
\mathbb{S}[\boldsymbol{L} u(x, t)+N u(x, t)+g(x, t)]
$$

Simplifying Eq. (10), to obtain:

$$
\begin{aligned}
& \mathbb{S}[u(x, t)]=\sum_{k=0}^{m-1} u^{k} f_{k}(x)+u^{\alpha} \mathbb{S}[\boldsymbol{L} u(x, t)+ \\
& \boldsymbol{N} u(x, t)+g(x, t)]
\end{aligned}
$$

Now, solution by standard Sumudu decomposition method is an infinite series as defined:

$$
u(x, t)=\quad \sum_{n=0}^{\infty} u_{n}(x,
$$

And the nonlinear term of [12] is decomposed as:

$$
\underset{(13)}{N u(x, t)} \quad=\quad \sum_{n=0}^{\infty} A_{n}(u)
$$

For some the Adomian polynomials $A_{n}$ of $u_{0}$, $u_{1}, \ldots, u_{n}, \ldots$ that are given by:

$A_{n}(u)=\left.\frac{1}{n !} \frac{d^{n}}{d \delta^{n}}\left[N\left(\sum_{i=0}^{\infty} \delta^{i} u_{i}\right)\right]\right|_{\delta=0}, \quad n=0,1$, $2, \ldots$

where $A_{n}$ for equation (14) can be obtained from the recurrent relation;

$$
A_{n}=\sum_{\substack{0<i<n \\ 0<j<n}} u_{i} \frac{\partial u_{j}}{\partial x}
$$

Now, substituting equations (12) and (13) into (11) to get:

$$
\begin{aligned}
& \mathbb{S}\left[\sum_{n=0}^{\infty} u_{n}(x, t)\right]= \\
& \sum_{k=0}^{m-1} u^{k} f_{k}(x)+u^{\alpha} \mathbb{S}\left[\boldsymbol{L} \sum_{n=0}^{\infty} u_{n}(x, t)+\right. \\
& \left.\sum_{n=0}^{\infty} A_{n}(u)+g(x, t)\right]
\end{aligned}
$$

For two sides of equation (15), the following algorithm is obtained

$$
\begin{aligned}
& \mathbb{S}\left[u_{0}(x, t)\right]=\sum_{k=0}^{m-1} u^{k} f_{k}(x) \\
& \mathbb{S}\left[u_{1}(x, t)\right]=u^{\alpha} \mathbb{S}\left[\boldsymbol{L} u_{0}(x, t)+A_{0}(u(x, t))\right. \\
& +g(x, t)] \\
& \mathbb{S}\left[u_{n+1}(x, t)\right]=u^{\alpha} \mathbb{S}\left[\boldsymbol{L} u_{n}(x, t)\right. \\
& \left.+A_{n}(u(x, t))\right], \quad n \geq 1 \text {. }
\end{aligned}
$$

Applying inverse ST on the equations above to get the following:

$$
\begin{aligned}
& u_{0}(x, t)=\mathbb{S}^{-1}\left(\sum_{k=0}^{m-1} u^{k} f_{k}(x)\right) \\
& u_{1}(x, t)=\mathbb{S}^{-1}\left(u ^ { \alpha } \mathbb { S } \left[\mathbf{L} u_{0}(x, t)+A_{0}(u(x, t))+\right.\right. \\
& g(x, t)])
\end{aligned}
$$

$u_{n+1}(x, t)=$

$\mathbb{S}^{-1}\left(u^{\alpha} \mathbb{S}\left[\boldsymbol{L} u_{n}(x, t)+A_{n}(u(x, t))\right]\right), n \geq 1$.

In the same way the rest of components of the decomposition solution can be found. Then the approximate solution is given by;

$$
u_{n}(x, t)=\sum_{j=0}^{n-1} u_{j}(x, t)
$$

Such that

$\lim _{n \rightarrow \infty} u_{n}(x, t)=u(x, t)$

Now applying ST method to solve the FDBE of the form:

$D_{t}^{\alpha} u+u u_{x}-u_{x x}+\lambda u=0, x \in \mathbb{R}, \quad \lambda, \alpha, t>0$ (18)

Now, solve equation (18) and take three cases:

Case (1) $0<\alpha<1, \lambda$ arbitrary positive real number, with IC $u(x, 0)=x$

Case (2) $0<\alpha<1, \lambda=1, u(x, 0)=x^{2}$

Case (3) $0<\alpha<2, \lambda=1, u(x, 0)=1$,

$u_{t}(x, 0)=\sin x$

\section{Status 1}

Let the value of $\alpha$ be extended to be any positive real number and solving the case $0<\alpha \leq 1$ and $\lambda=2$. If the IC $u(x, 0)=x$. Now, taking ST of equation (18) and the ST property is used with the IC, to get: $\mathbb{S}[u(x, t)]=x+u^{\alpha} \mathbb{S}\left[-u(x, t) \frac{\partial u}{\partial x}+\frac{\partial^{2}}{\partial x^{2}} u(x, t)-\right.$ $2 u(x, t)]$.

The inverse of ST as follows ;

$\sum_{n=0}^{\infty} u_{n}(x, t)=x+\mathbb{S}^{-1}\left[u^{\alpha} \mathbb{S}\left[-\sum_{n=0}^{\infty} A_{n}(u)+\right.\right.$

$\left.\left.\frac{\partial}{\partial x^{2}} \sum_{n=0}^{\infty} u_{n}(x, t)-2 \sum_{n=0}^{\infty} u_{n}(x, t)\right]\right]$

where $A_{n}(u)$ of (14) represents the nonlinear term $u u_{x}$. The first components polynomials are $A_{0}$ $=u_{0} u_{0 x}, \quad A_{1}=u_{0} u_{1 x}+u_{1} u_{0 x}, \quad A_{2}=u_{0} u_{2 x}+$ $u_{1} u_{1 x}+u_{2} u_{0 x}$ and so on. The recursive relation is given as:

$u_{0}(x, t)=x$

$u_{1}(x, t)=\mathbb{S}^{-1}\left[u^{\alpha} \mathbb{S}\left[-A_{0}(u)+\frac{\partial^{2}}{\partial x^{2}} u_{0}(x, t)-\right.\right.$

$\left.\left.2 u_{0}(x, t)\right]\right]$

$u_{n}(x, t)=$

$\mathbb{S}^{-1}\left[u^{\alpha} \mathbb{S}\left[\left[-A_{n-1}(u)+\frac{\partial^{2}}{\partial x^{2}} u_{n-1}(x, t)-\right.\right.\right.$

$\left.\left.2 u_{n-1}(x, t)\right]\right]$

Upon passing calculations, the following can be obtained

$u_{1}(x, t)=\frac{-3 x t^{\alpha}}{\Gamma(\alpha+1)}$

$u_{2}(x, t)=\frac{12 x t^{2 \alpha}}{\Gamma(2 \alpha+1)}$

$u_{3}(x, t)=\frac{-48 x t^{3 \alpha}}{\Gamma(3 \alpha+1)}-\frac{9 \Gamma(2 \alpha+1) x t^{3 \alpha}}{\Gamma(\alpha+1)^{2} \Gamma(3 \alpha+1)}$ 
$u_{4}(x, t)=\frac{192 x t^{4 \alpha}}{\Gamma(4 \alpha+1)}+\frac{36 \Gamma(2 \alpha+1) x t^{4 \alpha}}{\Gamma(\alpha+1)^{2} \Gamma(4 \alpha+1)}+$

$\frac{72 \Gamma(3 \alpha+1) x t^{4 \alpha}}{\Gamma(\alpha+1) \Gamma(2 \alpha+1) \Gamma(4 \alpha+1)}$

Etc.

Thus the solution of eq (18) is:

$$
\begin{aligned}
u(x, t)=\sum_{i=0}^{4} u_{i}(x, t) & \\
& =u_{0}(x, t)+u_{1}(x, t) \\
& +u_{2}(x, t)+u_{3}(x, t)+u_{4}(x, t)
\end{aligned}
$$

$\frac{9 \Gamma(2 \alpha+1) x t^{3 \alpha}}{\Gamma(\alpha+1)^{2} \Gamma(3 \alpha+1)}+\frac{192 x t^{4 \alpha}}{\Gamma(4 \alpha+1)}$

$$
+\frac{36 \Gamma(2 \alpha+1) x t^{4 \alpha}}{\Gamma(\alpha+1)^{2} \Gamma(4 \alpha+1)}+\frac{72 \Gamma(3 \alpha+1) x t^{4 \alpha}}{\Gamma(\alpha+1) \Gamma(2 \alpha+1) \Gamma(4 \alpha+1)}
$$

In Fig. 1 is 2 dimensional plot of the surface $u(x, t)$ for $\alpha=0.8,-1<x<2$ and $0<t<2$

u

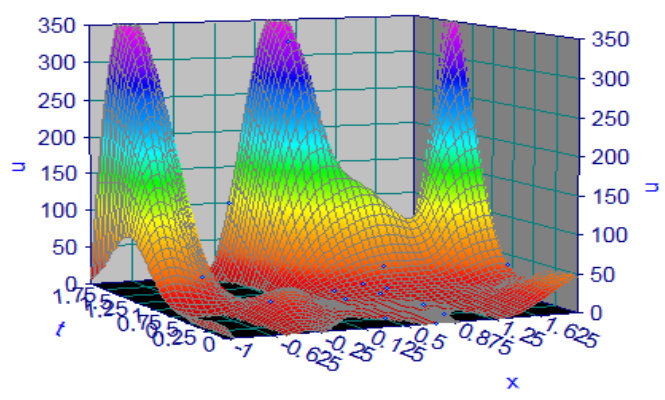

Figure 1. representing the solution when $\alpha=0.8$, $-1<x<2,0<t<2$ and $0<u<350$.

\section{Status 2}

If $0<\alpha \leq 1$ and $\lambda=1$, with the IC $u(x, 0)=x^{2}$. Taking ST of equation (18) and the ST property is used with the IC, consequently:

$$
\mathbb{S}[u(x, t)]=x^{2}+\quad u^{\alpha} \mathbb{S}\left[-u(x, t) \frac{\partial u}{\partial x}+\right.
$$

$\left.\frac{\partial^{2}}{\partial x^{2}} u(x, t)-u(x, t)\right]$

The inverse of ST is given as follows;

$\sum_{n=0}^{\infty} u_{n}(x, t)=x^{2}+\mathbb{S}^{-1}\left[u^{\alpha} \mathbb{S}\left[-\sum_{n=0}^{\infty} A_{n}(u)\right.\right.$

$\left.\left.+\frac{\partial}{\partial x^{2}} \sum_{n=0}^{\infty} u_{n}(x, t)-\sum_{n=0}^{\infty} u_{n}(x, t)\right]\right]$

where $A_{n}(u)$ of (14) represents the nonlinear term $u u_{x}$. The first components polynomials are $A_{0}$ $=u_{0} u_{0 x}, \quad A_{1}=u_{0} u_{1 x}+u_{1} u_{0 x}, \quad A_{2}=u_{0} u_{2 x}+$ $u_{1} u_{1 x}+u_{2} u_{0 x}$ and so on. The recursive relation is given as:

$$
\begin{aligned}
& u_{0}(x, t)=x^{2} \\
& u_{1}(x, t)=\frac{t^{\alpha}}{\Gamma(\alpha+1)}\left(2-x^{2}-2 x^{3}\right) \\
& u_{2}(x, t)=\frac{t^{2 \alpha}}{\Gamma(2 \alpha+1)}\left(-4-16 x+x^{2}+6 x^{3}+\right. \\
& \left.10 x^{4}\right) \\
& u_{3}(x, t)=\frac{t^{3 \alpha}}{\Gamma(3 \alpha+1)}\left(6+60 x+167 x^{2}-10 x^{3}-\right. \\
& \left.40 x^{4}-60 x^{5}\right)+\frac{\Gamma(2 \alpha+1) t^{3 \alpha}}{\Gamma(\alpha+1)^{2} \Gamma(3 \alpha+1)}(4 x \\
& \left.+12 x^{2}-2 x^{3}-10 x^{4}-12 x^{5}\right) \\
& u_{4}(x, t)=\frac{t^{4 \alpha}}{\Gamma(4 \alpha+1)}\left(328-132 x-827 x^{2}-\right. \\
& \left.778 x^{3}+90 x^{4}+300 x^{5}+150 x^{6}\right) \\
& +\frac{\Gamma(2 \alpha+1) t^{4 \alpha}}{\Gamma(\alpha+1)^{2} \Gamma(4 \alpha+1)}\left(24-16 x-144 x^{2}-\right. \\
& \left.286 x^{3}+20 x^{4}+72 x^{5}+84 x^{6}\right) \\
& +\frac{\Gamma(3 \alpha+1) t^{4 \alpha}}{\Gamma(\alpha+1) \Gamma(2 \alpha+1) \Gamma(4 \alpha+1)}(32-12 x- \\
& 108 x^{2}-204 x^{3}+40 x^{4}+132 x^{5} \\
& \left.+140 x^{6}\right)
\end{aligned}
$$

Etc.

Thus the solution of eq (18) is:

$$
\begin{aligned}
& u(x, t)=\sum_{i=0}^{4} u_{i}(x, t)=u_{0}(x, t)+u_{1}(x, t)+ \\
& \begin{aligned}
u_{2}(x, t)+u_{3}(x, t)+u_{4}(x, t) \\
=x^{2}+\frac{t^{\alpha}}{\Gamma(\alpha+1)}\left(2-x^{2}-2 x^{3}\right)+ \\
\frac{t^{2 \alpha}}{\Gamma(2 \alpha+1)}\left(-4-16 x+x^{2}+6 x^{3}+10 x^{4}\right) \\
+\frac{t^{3 \alpha}}{\Gamma(3 \alpha+1)}\left(6+60 x+167 x^{2}-10 x^{3}-\right. \\
\left.40 x^{4}-60 x^{5}\right)+\frac{\Gamma(2 \alpha+1) t^{3 \alpha}}{\Gamma(\alpha+1)^{2} \Gamma(3 \alpha+1)}(4 x+ \\
\left.12 x^{2}-2 x^{3}-10 x^{4}-12 x^{5}\right)+ \\
\frac{t^{4 \alpha}}{\Gamma(4 \alpha+1)}\left(328-132 x-827 x^{2}-778 x^{3}\right.
\end{aligned}
\end{aligned}
$$

$$
\begin{aligned}
& \left.+90 x^{4}+300 x^{5}+150 x^{6}\right)+\frac{\Gamma(2 \alpha+1) t^{4 \alpha}}{\Gamma(\alpha+1)^{2} \Gamma(4 \alpha+1)}(24- \\
& 16 x-144 x^{2} \\
& \left.\quad-286 x^{3}+20 x^{4}+72 x^{5}+84 x^{6}\right)+ \\
& \frac{\Gamma(3 \alpha+1) t^{4 \alpha}}{\Gamma(\alpha+1) \Gamma(2 \alpha+1) \Gamma(4 \alpha+1)}(32-12 x \\
& \quad-108 x^{2}-204 x^{3}+40 x^{4}+132 x^{5}+ \\
& \left.140 x^{6}\right)
\end{aligned}
$$

In Fig. 2 is 2 dimensional plot of the surface $u(x, t)$ for $\alpha=0.8, \quad 0<x<1$ and $0<t<1$ 


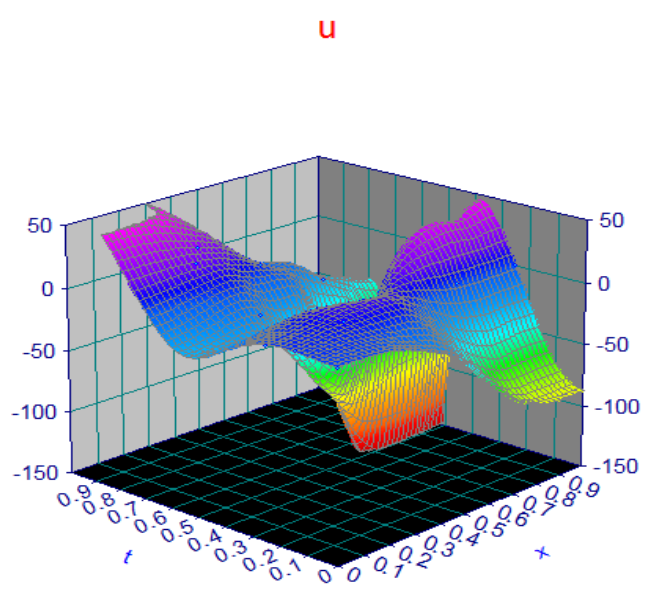

Figure 2. representing the solution when $\alpha=0.8$, $0<x<1,0<t<1$ and $-150<u<50$.

\section{Status 3}

If $0<\alpha \leq 2$ and $\lambda=1$, with the ICs $u(x, 0)=1$, $u_{t}(x, 0)=\sin x$. with the IC $u(x, 0)=x^{2}$. Taking ST of equation (18) and the ST property is used with the IC, then:

$\mathbb{S}[u(x, t)]=1+t \sin x+u^{\alpha} \mathbb{S}\left[-u(x, t) \frac{\partial u}{\partial x}+\right.$ $\left.\frac{\partial^{2}}{\partial x^{2}} u(x, t)-u(x, t)\right]$

The inverse of ST is given as follows;

$\sum_{n=0}^{\infty} u_{n}(x, t)=$

$1+t \sin +\mathbb{S}^{-1}\left[u^{\alpha} \mathbb{S}\left[-\sum_{n=0}^{\infty} A_{n}(u)+\right.\right.$

$\left.\left.\frac{\partial}{\partial x^{2}} \sum_{n=0}^{\infty} u_{n}(x, t)-\sum_{n=0}^{\infty} u_{n}(x, t)\right]\right]$

where $A_{n}(u)$ of (14) represents the nonlinear term $u u_{x}$. The first components polynomials are $A_{0}$ $=u_{0} u_{0 x}, \quad A_{1}=u_{0} u_{1 x}+u_{1} u_{0 x}, \quad A_{2}=u_{0} u_{2 x}+$ $u_{1} u_{1 x}+u_{2} u_{0 x}$ and so on. The recursive relation is given as:

$$
\begin{aligned}
& u_{0}(x, t)=1+t \sin x \\
& u_{1}(x, t)=\frac{-t^{\alpha}}{\Gamma(\alpha+1)}-\frac{2 \sin x t^{\alpha+1}}{\Gamma(\alpha+2)}-\frac{\cos x t^{\alpha+1}}{\Gamma(\alpha+2)}- \\
& \frac{\cos x \sin x t^{\alpha+2}}{\Gamma(\alpha+3)} \\
& u_{2}(x, t)=\frac{t^{2 \alpha}}{\Gamma(2 \alpha+1)}+\frac{4 \cos x t^{2 \alpha+1}}{\Gamma(2 \alpha+2)}+\frac{3 \sin x t^{2 \alpha+1}}{\Gamma(2 \alpha+2)}+ \\
& \frac{5 \cos x \sin x t^{2 \alpha+2}}{\Gamma(2 \alpha+3)}+\frac{\left(\cos ^{2} x-\sin ^{2} x\right) t^{2 \alpha+2}}{\Gamma(2 \alpha+3)} \\
& +\frac{4 \Gamma(\alpha+3) \sin x \cos x t^{2 \alpha+2}}{\Gamma(\alpha+2) \Gamma(2 \alpha+3)}- \\
& \frac{\Gamma(\alpha+3)\left(\sin ^{2} x-\cos ^{2} x\right) t^{2 \alpha+2}}{\Gamma(\alpha+2) \Gamma(2 \alpha+3)}+\frac{\Gamma(\alpha+2) \cos x t^{2 \alpha+1}}{\Gamma(\alpha+1) \Gamma(2 \alpha+2)} \\
& \quad+\frac{2 \Gamma(\alpha+4) \cos ^{2} x \sin x t^{2 \alpha+3}}{\Gamma(\alpha+3) \Gamma(2 \alpha+4)}
\end{aligned}
$$

Etc.

Thus the solution of eq (18) is:

$$
\begin{aligned}
& u(x, t)=\sum_{i=0}^{2} u_{i}(x, t)=u_{0}(x, t)+u_{1}(x, t)+ \\
& u_{2}(x, t) \\
& =1+t \sin x-\frac{t^{\alpha}}{\Gamma(\alpha+1)}-\frac{2 \sin x t^{\alpha+1}}{\Gamma(\alpha+2)}- \\
& \frac{\cos x t^{\alpha+1}}{\Gamma(\alpha+2)}-\frac{\cos x \sin x t^{\alpha+2}}{\Gamma(\alpha+3)}+\frac{t^{2 \alpha}}{\Gamma(2 \alpha+1)} \\
& +\frac{4 \cos x t^{2 \alpha+1}}{\Gamma(2 \alpha+2)}+\frac{3 \sin x t^{2 \alpha+1}}{\Gamma(2 \alpha+2)}+ \\
& \frac{5 \cos x \sin x t^{2 \alpha+2}}{\Gamma(2 \alpha+3)}+\frac{\left(\cos ^{2} x-\sin ^{2} x\right) t^{2 \alpha+2}}{\Gamma(2 \alpha+3)}+ \\
& \frac{4 \Gamma(\alpha+3) \sin x \cos x t^{2 \alpha+2}}{\Gamma(\alpha+2) \Gamma(2 \alpha+3)}- \\
& \frac{\Gamma(\alpha+3)\left(\sin ^{2} x-\cos ^{2} x\right) t^{2 \alpha+2}}{\Gamma(\alpha+2) \Gamma(2 \alpha+3)}+\frac{\Gamma(\alpha+2) \cos x t^{2 \alpha+1}}{\Gamma(\alpha+1) \Gamma(2 \alpha+2)} \\
& +\frac{2 \Gamma(\alpha+4) \cos ^{2} x \sin x t^{2 \alpha+3}}{\Gamma(\alpha+3) \Gamma(2 \alpha+4)}
\end{aligned}
$$

In Fig. 3 is 2 dimensional plot of the surface $u(x, t)$ for $\alpha=0.8, \quad 0<x<3$ and $0<t<5$

$$
\text { u }
$$

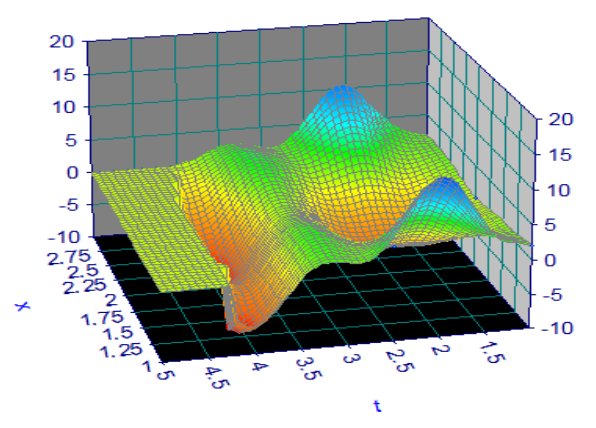

Figure 3. representing the solution when $\alpha=0.8$, $1<x<3,1<t<5$ and $-10<u<20$.

\section{Conclusions:}

The primary objective of this work is to suggest an effective technique for solving nonlinear fractional BE. This method was a power tool to solve various kinds of nonlinear problems. The time-fractional BEs are chosen with the ICs to illustrate the proposition technique. In this paper, using different types of figure to solve the Burgers' equations by using the Sumudu transform method. To solve the linear and nonlinear partial differential equations, ST method can be used and it can be applied to one and 2-diimensional fractional equations with accurately and successfully.

\section{Authors' declaration:}

- Conflicts of Interest: None.

- We hereby confirm that all the Figures and Tables in the manuscript are mine ours. Besides, the Figures and images, which are not mine ours, 
have been given the permission for republication attached with the manuscript.

- Ethical Clearance: The project was approved by the local ethical committee in University of AlEsraa.

\section{References:}

1. AL-Khaled K. Numerical solution of time-fractional partial differential equations using sumudu decomposition method. Rom. J. Phys. 2015; 60( 1-2): 99-110.

2. Pandey K, Verma L. A note on Crank Nicolson scheme for burgers Equation. Appl Math. 2011; 2: 883-889.

3. Bateman H. Some recent researches on the motion of fluids. Mon. Weather Rev. 1915; 43(4):163-170.

4. Burger J M. A mathematical model illustrating the theory of turbulence. Adv Appl Mech. 1948; 1: 171199.

5. Cole J D. On a quasi-linear parabolic equation occurring in aerodynamics. Quart Appl Math. 1951; 9(3): 225-236.

6. Gorguis A. A comparison between Cole-Hopf transformation and decomposition method for solving Burgers equations. Appl Math Comput. 2006; 173(1): 126-136.

7. Momani S. Non-perturbative analytical solutions of the space- and time-fractional Burgers equations. Chaos, Solitons Fractals. 2006; 28(4): 930-937.

8. Blazer J, Aminikhah H. Exact and numerical solutions for non-linear Burger's equation by VIM. Mathematical and Computing Modeling, 2009. 49( 78): 1394-1400.
9. AL-Azawi S N, Salih M. Using Bernoulli equation to solve Burger's equation. Baghdad Sci J; 2014. 11(2): 202-206.

10. Watugala G K. Sumudu transform: a new integral transform to solve differential equations and control engineering problems. Int J Math Educ Sci Technol. 1993; 24(1): 35-43.

11. Rathore S, Kumar D, Singh J, Gupta S. Homotopy Analysis sumudu transform method for nonlinear equations . Int. J. Industrial Mathematics. 2012; 4(4): 301-314.

12. Kurulay M. The Approximate and Exact Solutions of the Space- and Time-Fractional Burgers Equations. Ijrras. 2010; 3(3): 257-263.

13. Javidi M, Ahmed B. Numerical Solution of Fractional Partial Differential Equations by Numerical Laplace Inversion Technique. Adv Differ Equ-ny. 2013; 1 (375): 1-18.

14. Çetinkaya A, Kıymaz O, Çamlı J. Solutions of nonlinear PDE's of fractional order with generalized differential transform method. Int Math Forum. 2011; 6(1): 39-47.

15. Karbalaie A, Montazeri M M, Muhammed H H. Exact Solution of time-fractional partial differential equations using sumudu transform. Wseas Trans Math. 2014; 13: 142-151.

16. Mahdy M A S, Mohamed A S, Mtawa A A. Implementation of the homotopy perturbation sumudu transform method for solving Klein-Gordon equation. Applied Mathematics, 2015. 6: 617-628.

17. Akpan I. P. Adomian decomposition approach to the solution of the Burger's equation. Am J Comput Math. 2015; 5(3): 329-335.

\title{
حل معادلات Burgers Damped تقريبًا باستخام طريقة التحويل ( ST) (Sumudu)
} \\ في هذا البحث ، يتم حل معادلة بيركر دامبت الكسرية من الصيخة

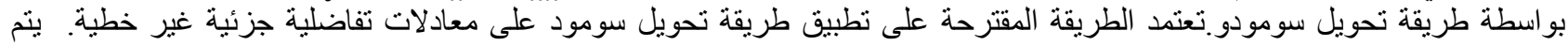

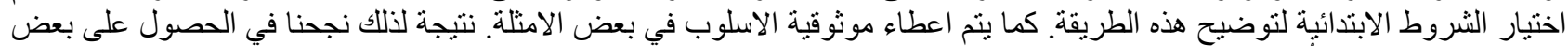 \\ الحلول التقريبية ـ اخيرأ، يستخدم برنامج منحني ثناثي الابعاد لرسم الاشيكال. \\ الكلمات المفتاحية: تحويل Sumudu ، حساب التفاضل و التكامل الكسري، مشتقة Caputo ، معادلة damped Burger الكسرية
}

